Original Research Paper

\title{
GoogleNet CNN Neural Network towards Chest CT- Coronavirus Medical Image Classification
}

\author{
${ }^{1}$ Nesreen Alsharman and ${ }^{2}$ Ibrahim Jawarneh \\ ${ }^{1}$ Department of Computer Science, WISE, Amman, Jordan \\ ${ }^{2}$ Department of Math, AHU, Ma'an, Jordan
}

Article history

Received: 11-04-2020

Revised: 07-05-2020

Accepted: 14-05-2020

Corresponding Author:

Nesreen Alsharman

Department of Computer

Science, WISE, Amman,

Jordan

Email: nesreen.alsharman@wise.edu.jo

\begin{abstract}
In the end of the year 2019 and the beginning of the year 2020, the world was overwhelmed by a medical pandemic that was not previously seen which is known Covid-19 (Coronavirus). Coronavirus (CoV) is a large family of viruses that cause illness ranging from the common cold to more severe diseases such as Middle East Respiratory Syndrome (MERS-CoV) and Severe Acute Respiratory Syndrome (SARS-CoV). This paper aims to improve the accuracy of detection for CT-Coronavirus images using deep learning for Convolutional Neural Networks (CNNs) that helps medical staffs for classification chest CT- Coronavirus medical image in early stage. Deep learning is successfully used as a tool for machine learning, where the CNNs are capable of automatically extracting and learning features medical image dataset. This research retrains GoogleNet CNN architecture over the COVIDCT-Dataset for classification CT- Coronavirus image. In this research, COVIDCT-Dataset contains 349 CT images containing clinical findings of COVID-19. The validation accuracy of retraining GoogleNet is $82.14 \%$ where elapsed time is $74 \mathrm{~min}$ and $37 \mathrm{sec}$.
\end{abstract}

Keywords: GoogleNet, CNN, CT-Coronavirus Medical Image, Image Classification

\section{Introduction}

COVID-19 pandemic is a new strain discovered in 2019 and not previously known in humans. Coronaviruses are zoonotic which mean that they are spread between animals and humans. Specific studies showed that SARS-CoV was transmitted to humans from civet cats and MERS-CoV from dromedary camels (WHO, 2020). Many identified Coronavirus occur in animals not yet infected by humans. Respiratory symptoms, fever, cough, shortness of breath and trouble breathing are typical signs of infection. In more serious cases, pneumonia, extreme acute respiratory syndrome, kidney failure and even death may result from infection. Standard guidelines for preventing spread of infection include daily hand washing, mouth and nose covering while coughing and sneezing, thorough cooking of meat and eggs. Avoid direct contact with someone who has respiratory problems such as coughing and sneezing (Chung et al., 2019). CT scan images of these patients' chests that were returned by medical team revealed certain common features including, consolidation features and ground glass opacities over the lungs. The team also noted the number of lobes of the lungs that were affected with ground glass opacities and consolidation and the degree of involvement of each of these lobes of the lungs. Based on these the team also looked at the overall total severity scores. They also noted absence of nodules in the lungs and fluid in the pleural cavity (pleural effusion). There was involvement of some of the lymph nodes of the thorax and these were abnormal in size and shape, they wrote. Some of the patients also had underlying lung diseases such as fibrosis and emphysema. These features were all recorded for the patients with the Coronavirus infections. The team found some additional features such as crazy paving patterns and distribution of the changes in the peripheral parts of the lungs (Chung et al., 2019).

In the past, between the 1970s and 1990s, the analysis of medical image was done by two traditional ways: (1) The mathematical modeling (fitting lines, ellipses and circles) to construct the compound rulebased systems for solving the particular tasks and (2) low-level pixel processing (edge and line detector filters, region growing) using sequential application. At the beginning of 2000, the supervised techniques machine learning was developed as training data system for medical image analysis. The supervised techniques for 
medical image analysis could be used for different tasks such as segmentation as active shape models, extracting new data from training data that called atlas methods and extracting new feature that use by statistical classifier that is popular approach of many successful commercially available medical image analysis systems (Litjens et al., 2017).

The extraction of discriminant features from the medical images is an important and crucial step in the design of supervised techniques. Human researchers are still doing this process and, as such, one speaks of systems with handcrafted features. Since the proposal of a fast learning algorithm for deep layer networks. The deep learning techniques have drawn ever-increasing research interests because of their inherent capability of overcoming the drawback of traditional algorithms dependent on hand-designed features. Deep learning approaches have also been found to be suitable for big data analysis with successful applications to computer vision, medical image analysis, speech recognition, recommendation systems and natural language processing. The Deep learning success in perceptual tasks is largely due to its automation of the process of feature extraction engineering: Hierarchical feature extractors are learned from data in an end-to-end manner, rather than manually designed.

Deep learning has made tremendous progress in a variety of tasks over the past few years, such as image recognition, speech recognition and machine translation. Novel neural network architectures are one key thing for this development. Currently employed architectures have mostly been developed manually by human experts, which is error prone process and a time-consuming. Because of this, there is growing interest in automated neural network architecture search methods (Wistuba et al., 2019).

The special kind of deep learning neural network is the Convolutional Neural Networks (CNN) that have rapidly become an efficient method for classification medical images. CNNs are one of the best learning algorithms for understanding image content and have shown exemplary performance in classification, detection, image segmentation and retrieval related tasks (Liu et al., 2019; Ciresan et al., 2012). This research retrained GoogleNet CNN neural network that helps chest CT-Coronavirus medical image classification in early stage.

\section{Chest CT- Coronavirus Medical Image}

The study (Li and Xia, 2020) was to determine the misdiagnosis rate of radiologists for Coronavirus disease 2019 (COVID-19) and evaluate the performance of chest $\mathrm{CT}$ in the diagnosis and management of COVID-19. The CT features of COVID-19 are reported and compared with the CT features of other viruses to familiarize radiologists with possible $\mathrm{CT}$ patterns. Figure 1 shows an example of CT-Coronavirus medical image. The Deep learning is a computerized technique that success in perceptual tasks is largely due to its automation of the process of feature extraction engineering: hierarchical feature extractors are learned from data in an end-to-end manner, rather than manually designed.

\section{Related Work}

Neural networks have been successfully used to many medical image processing and analysis problems in recent works because the newest neural network architectures often add connections and additional operations to the standard architecture that enable training deeper networks (Albarqouni et al., 2016; Bao and Chung, 2018). In this section, four neural network architectures are covered (Schmidhuber, 2014). The first architecture is stacked layer that the multiple, monodirectional such as feed forward neural network architectures (Møller, 1993) layers are stacked in such a way, this model is referred to as a Multi-Layered Perception (MLP), where the intermediate layers are typically known as hidden layers as they are not directly observed (in contrast to the in- and output layers).

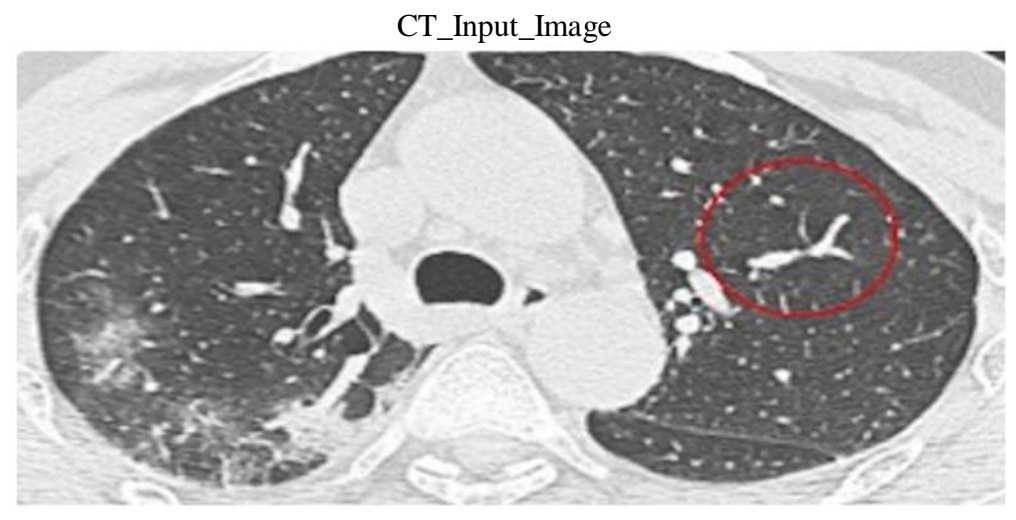

Fig. 1: Example of CT-Coronavirus Medical Image (Zhao et al., 2020) 
The second architecture is Deep Neural Network (DNN) (Szegedy et al., 2013) where the network has many layers. layer-by-layer in an unsupervised manner (pretraining), followed by supervised fine-tuning of the stacked network, could result in excellent pattern recognition tools. Two common DNN architectures trained in such a way are Deep Belief Networks (DBNs) and (SAEs) stacked auto encoders. The third and fourth architectures are Convolutions Neural Networks (CNNs) (Bengio et al., 2007) and Recurrent Neural Networks (RNNs) (Prokhorov et al., 2002) that work for image analysis. The CNNs and RNNs are an architectures alternative architectures strategy that are used to train an entire deep network end-to-end in a supervised fashion.

The oldest neural network architecture is perception the which was developed back in 1958 (Rosenblatt, 1958; Friedberg, 1958). This is the simplest neural network, as well. The perception was invented by Frank Rosenblatt and laid the foundations for the fundamentals of neural networks. The simplest perception neural network only has one neuron. It takes $\mathrm{n}$ inputs and multiplies them by weights that correspond. It only computes one output. Due to its lack of sophistication, it fails in that it can process medical image analysis data. From the other hand this architecture of neural network works only with one degree of complexity. Also, this type allows us for understanding the human brain and scaling up for more advanced neural networks. Then the MLPs architecture is also perception neural network with adding more layer. Feed forward neural network (Møller, 1993) is an example MLP. It works by passing numerical data into the networks and it continues forward having many operations done to it. For getting the right operations such that any input given will always produce a desired output requires training. Training is essentially finding what yields the best results and applying them to the network. This type works with Computer Vision and Natural Language Processing and it does not work for medical image analysis.

One of the best neural network learning algorithms are CNNs that were generated after DNNs because DNNs were considered hard to train efficiently and only gained popularity in 2006 (Hinton et al., 2006; Hinton and Salakhutdinov, 2006; Bengio et al., 2007). CNNs could understand image content and have demonstrated exemplary success in image classification, identification and image segmentation related tasks for retrieval (Liu et al., 2019). A typical CNN architecture generally comprises of alternate layers of convolution and pooling followed by one or more fully connected layers at the end. In some cases, fully connected layer is replaced with global average pooling layer. In addition to different mapping functions, different regulatory units such as batch normalization and dropout are also incorporated to optimize CNN performance (O’Shea and Nash, 2015).

\section{Proposed Method}

The proposed method consists of three main stages that are Preprocessing, Retraining GoogleNet CNN and Classification stages. Figure 2 shows the proposed method.

First, the CT- Coronavirus image has a lot of redundant information when it is captured. To get an acceptable and accurate image, the input CT-Coronavirus images need to be preprocessed where the CT-Coronavirus image can be done using some process such as the image enhancement, Normalization, Filtering, Noise reduction, Binarization and Thinning. In this research, the binarization is applied since it is the most preprocessing step that used before retaining image dataset. In addition, the preprocessing in this research contains a converting input image and dataset store images from 2D Grayscale to 3D Color:

\section{- 2D Grayscale to 3D Color}

The main idea to convert from 2D Grayscale to 3D color is that the CNNs just accept the image with 3D color so that the following MAT LAB function is used for converting.

augmented TrainingSet $=$ augmentedImageDatastore (imageSize,trainingSet,

'ColorPreprocessing','gray2rgb'); augmentedTestSet=augmentedImageDatastore (imageSize, testSet, 'ColorPreprocessing', 'gray2rgb');

- Binarization: The separation of the object and background is known as Binarization. In this research, imbinarize (I) function using MATLAB generates a binary image from $2 \mathrm{D}$ grayscale or $3 \mathrm{D}$ color image I by replacing all values above a globally determined threshold with $1 \mathrm{~s}$ and setting all other values to 0s. By default, imbinarize uses Otsu's method, which chooses the threshold value to minimize the intraclass variance of the thresholder black and white pixel. Imbinarize (1) uses a 256-bin image histogram to compute Otsu's threshold.
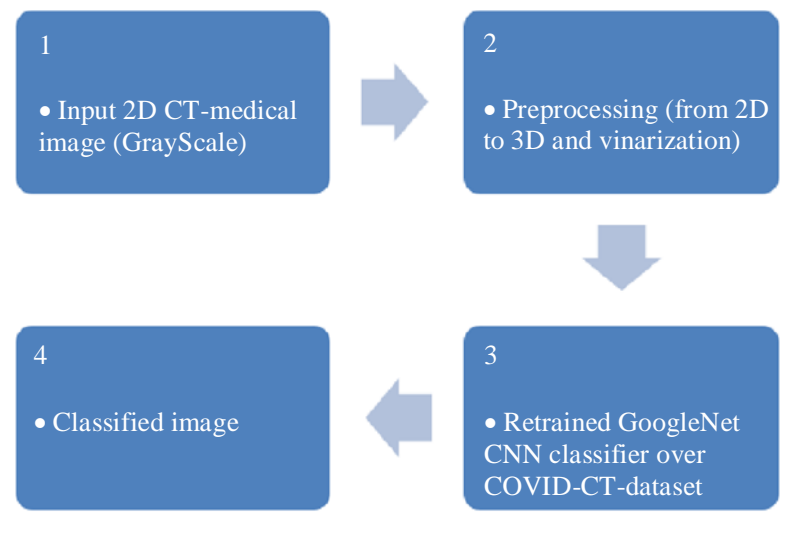

Fig. 2: Proposed method 
Second, the GoogleNet CNN architecture classifier is retrained over COVID-CT-Dataset (Zhao et al., 2020). GoogleNet is known as Inception-V1 where the main goal of this architecture was to get high accuracy and reduce computational cost (Szegedy et al., 2015). It provided a new concept of inception block in CNN which incorporates multi-scale convolutional transformations using split, transform and merge idea. This block encapsulates filters of different sizes $(1 \times 1$, $3 \times 3$ and $5 \times 5$ ) to capture spatial information at different scales (both at fine and coarse grain level). In GoogleNet, convolutional layers are replaced in small blocks like the idea of substituting each layer with micro $\mathrm{NN}$ as proposed in Network in Network (NIN) architecture (Lin et al., 2013). The exploitation of the idea of split, transform and merge by GoogleNet, helped in addressing a problem related to the learning of diverse types of variations present in the same category of different images. In addition to the improvement in learning capacity, GoogleNet makes the CNN parameter more efficiently.

Third, after retaining GoogleNet, any new input COVID-CT image could be classified.

\section{Experiment Result}

This section describes an experiment that conducted to test the proposed model for GoogleNet CNN neural network towards chest CT- Coronavirus medical image classification. The proposed model implementation runs using MATLAB environment with a PC containing 4 GB of RAM, 4 Intel cores, i5 (2.0 GHz each).

In our experiment, we used the COVID-CTDataset that has $349 \mathrm{CT}$ images containing clinical findings of COVID-19 (Zhao et al., 2020). Figure 3 shows the results of retraining GoogleNet over COVID-CT-Dataset, figure 4 shows a sample of COVID-CT-Dataset that used for retraining GoogleNet. The validation accuracy of retraining GoogleNet is $82.14 \%$ where elapsed time is $74 \mathrm{~min}$ and $37 \mathrm{sec}$, the number of epochs is 8 , the number of iterations is 592 and the number of iterations per epoch are 74 . The reason for this elapsed time is adding more layers in CNNs which leads to extract more features that indicate high accurate results. This accuracy could be achieved with more computation time. The main goal of the GoogleNet architecture is to achieve accuracy, so that the motivation for the GoogleNet is creating inception CNN module to make a deeper $\mathrm{CNN}$ by adding 22 layers. The training options in the experiment is as the following:

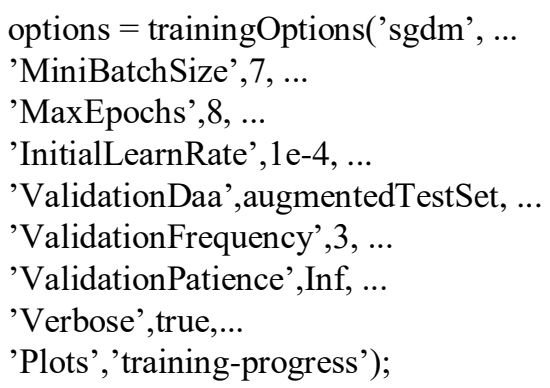

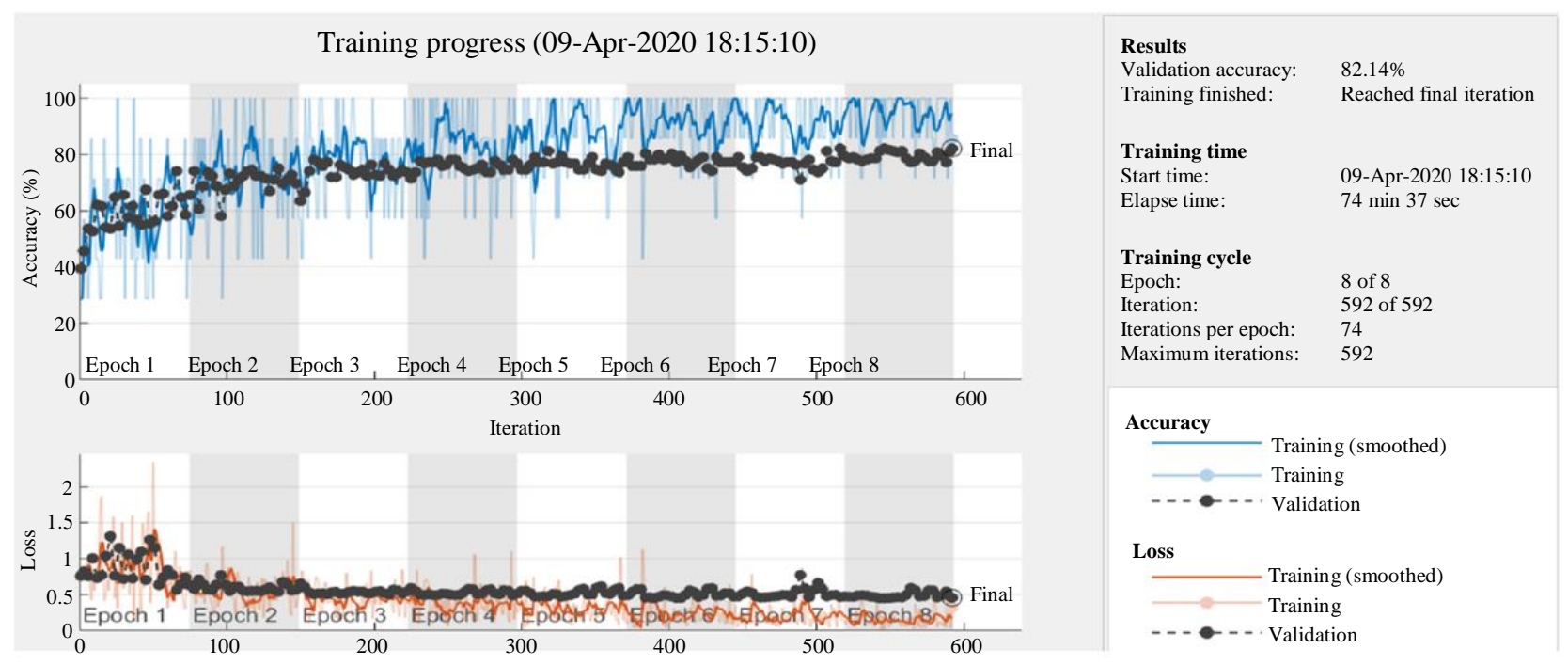

Fig. 3: Results of Retraining GoogleNet over COVID-CT-dataset 

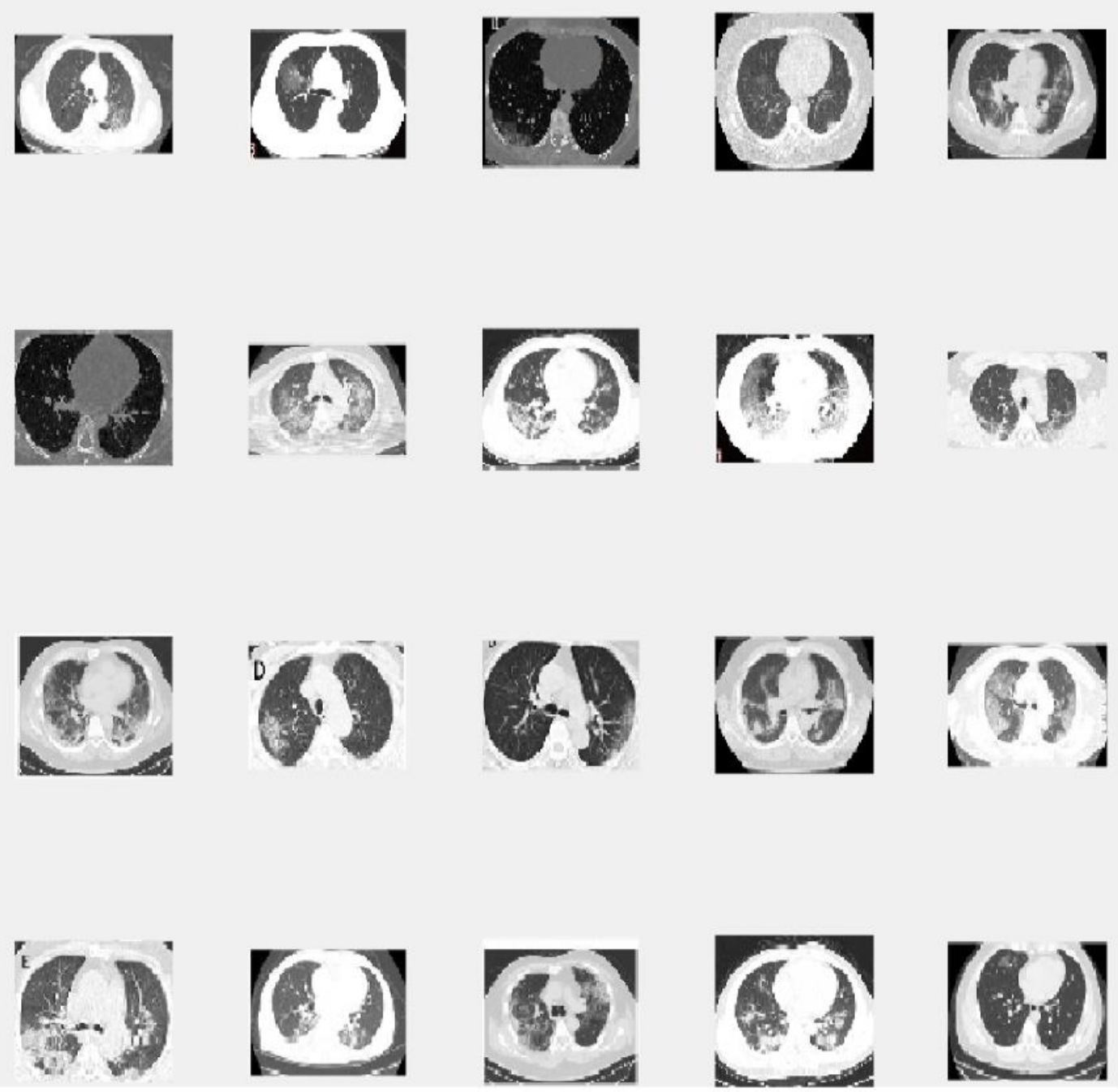

Fig. 4: Sample of COVID-CT-dataset that used for retraining GoogleNet

\section{Conclusion}

This paper discuses artificial intelligence with computer science for early medical diagnosis by applying deep learning techniques to extract features from chest CT- Coronavirus imaging that helps medical staff to classify the image in early stage. The 2019 novel Coronavirus exhibits hallmark features of chest CTCoronavirus imaging which are beneficial for the radiologist to identify and diagnose this new global health emergency. So that this research suggests GoogleNet as one possible deep learning computer solution for chest CT- Coronavirus image classification in early stage. In the future, we suggest retraining different CNNs architectures with different training options in the experiment to increase validation accuracy from $82.14 \%$ to $90 \%$ or above. In addition, we suggest doing medical image segmentation as preprocessing step.

\section{Acknowledgment}

The authors are indebted to editor and reviewers for their support and for their helpful comments from which this article has benefited much. They thank their universities WISE and AHU for their support.

\section{Author's Contributions}

All authors have equally contributed to the final version of the manuscript.

\section{Ethics}

This article is original and contains unpublished material. The corresponding author confirms that all authors have read and approved the manuscript and there are no ethical issues involved. 


\section{References}

Albarqouni, S., C. Baur, F. Achilles, V. Belagiannis and S. Demirci et al., 2016. Aggnet: Deep learning from crowds for mitosis detection in breast cancer histology images. IEEE Trans. Medical Imag., 35: 1313-1321. DOI: 10.1109/TMI.2016.2528120

Bao, S. and A.C.S. Chung, 2018. Multi-scale structured CNN with label consistency for brain MR image segmentation. Comput. Meth. Biomechan. Biomed. Eng.: Imag. Visualizat., 6: 113-117.

DOI: 10.1080/21681163.2016.1182072

Bengio, Y., P. Lamblin, D. Popovici and H. Larochelle, 2007. Greedy Layer-Wise Training of Deep Networks. In: Advances in Neural Information Processing Systems, Scholkopf, B., J.C. Platt and T. Hoffman (Eds.), MIT Press, pp: 153-160.

Chung, M., A. Bernheim, X. Mei, N. Zhang and M. Huang et al., 2019. CT imaging features of 2019 novel coronavirus (2019-nCoV). Radiology, 295: 202-207. DOI: 10.1148/radiol.2020200230

Ciresan, D., A. Giusti, L.M. Gambardella and J. Schmidhuber, 2012. Deep neural networks segment neuronal membranes in electron microscopy images. Proceedings of the 25th International Conference on Neural Information Processing Systems, (IPS' 12), ACM, Curran Associates, Inc., pp: 2843-2851.

Friedberg, R.M., 1958. A learning machine: Part I. IBM J. Res. Dev., 2: 2-13. DOI: 10.1147/rd.21.0002

Hinton, G.E. and R.R. Salakhutdinov, 2006. Reducing the dimensionality of data with neural networks. Science, 313: 504-507.

DOI: $10.1126 /$ science. 1127647

Hinton, G.E., S. Osindero and Y.W. The, 2006. A fast learning algorithm for deep belief nets. Neural Comput., 18: 1527-1554. DOI: 10.1162/neco.2006.18.7.1527

Li, Y. and L. Xia, 2020. Coronavirus disease 2019 (covid-19): Role of chest CT in diagnosis and management. Am. J. Roentgenol.

DOI: 10.2214/AJR.20.22954

Lin, M., Q. Chen and S. Yan, 2013. Network in network. CoRR.

Litjens, G., T. Kooi, B.E. Bejnordi, A.A.A. Setio and F. Ciompi, 2017. A survey on deep learning in medical image analysis. Medical Image Anal., 42: 60-88. DOI: 10.1016/j.media.2017.07.005

Liu, X., Z. Deng and Y. Yang, 2019. Recent progress in semantic image segmentation. Artificial Intell. Rev., 52: 1089-1106. DOI: 10.1007/s10462-018-9641-3

Møller, M.F., 1993. Exact calculation of the product of the Hessian matrix of feed-forward network error functions and a vector in $\mathrm{O}(\mathrm{N})$ time. Technical Report PB-432, Computer Science Department, Aarhus University, Denmark.
O'Shea, K. and R. Nash, 2015. An introduction to convolutional neural networks. CoRR.

Prokhorov, D.V., L.A. Feldkamp and I.Y. Tyukin, 2002. Adaptive behavior with fixed weights in RNN: An overview. Proceedings of the IEEE International Joint Conference on Neural Networks, May 12-17, IEEE Xplore Press, Honolulu, HI, USA, pp: 2018-2023. DOI: 10.1109/IJCNN.2002.1007449

Rosenblatt, F., 1958. The perceptron: A probabilistic model for information storage and organization in the brain. Psychol. Rev., 65: 386-386.

DOI: $10.1037 / \mathrm{h} 0042519$

Schmidhuber, J., 2014. Deep learning in neural networks: An overview. Neural Netw., 61: 85-117. DOI: 10.1016/j.neunet.2014.09.003

Szegedy, C., A. Toshev and D. Erhan, 2013. Deep neural networks for object detection. Proceedings of the Advances in Neural Information Processing Systems, (IPS’ 13), pp: 2553-2561.

Szegedy, C., W. Liu, Y. Jia, P. Sermanet and S. Reed et al., 2015. Going deeper with convolutions. Proceedings of the IEEE Conference on Computer Vision and Pattern Recognition, Jun. 7-12, IEEE Xplore Press, Boston, MA, USA. DOI: 10.1109/CVPR.2015.7298594

WHO, 2020. World health organization.

Wistuba, M., A. Rawat and T. Pedapati, 2019. A survey on neural architecture search. J. Machine Learn. Res.

Zhao, J., Y. Zhang, X. He and P. Xie, 2020. Covid-CTdataset: A CT scan dataset about covid-19. arXiv preprint arXiv:2003.13865. 